\title{
ABSOLUTE PROPER MOTIONS AND SPACE VELOCITIES OF GLOBULAR CLUSTERS
}

\author{
H. -J. Tucholke $e^{1,2}$, P. Brosche ${ }^{1}$ and M. Geffert ${ }^{1}$ \\ Observatorium Hoher List 1 \\ University of Munster 2
}

\begin{abstract}
Proper motions of globular clusters referred to extragalactic background objects provide absolute proper motions leading to space velocities. Some results from the Bonn Observatory proper motion program for galactic globular clusters are shown. Reference stars from the Lick program linked to galaxies are used. Low orbital angular momenta for NGC 4147, NGC 5466 and NGC 6218 were detected. In a further program, proper motions of 47 Tuc and NGC 362 are currently being measured relative to the background of the SMC.
\end{abstract}

\section{INTRODUCTION}

Absolute proper motions of globular clusters are important for the determination of the galactic halo dynamics, since, combined with radial velocities, they yield space velocities. In order to proceed from relative to absolute proper motions, one generally models the motions of the field stars by the superposition of solar motion and galactic rotation. The validity of this model has been questioned because of large unexplained components in the proper motions of fundamental stars (Brosche and Schwan 1981, 1986). An independent way to link globular cluster proper motions to an absolute reference frame is followed in Bonn: The cluster proper motions are referred to extragalactic objects, which should show zero proper motion within the measuring accuracy.

\section{THE BONN OBSERVATORY PROPER MOTION PROGRAM}

The following globular clusters are observed in the Bonn proper motion program: NGC 4147, NGC 5024, NGC 5272, NGC 5466, NGC 5904, NGC 6205, NGC 6218, NGC 6254, NGC 6341, NGC 6779, NGC 6934, NGC 7078 and NGC 7089. 01d and new plates with epoch differences from 60 to 70 years were taken with the double refractor of the Bonner Sternwarte. The error of the cluster mean motion is $0: 05$ to $0 ! 10 / 100 \mathrm{yr}$. For clusters at sufficiently high galactic latitude, stars from the Lick program with proper motions measured relative to galaxies are used as reference stars. In the future HIPPARCOS, whose system will be tied to an extragalactic inertial frame, will observe some reference stars in the field of each cluster. 
Up to now results were published for NGC 5466 (Brosche et al. 1983) and NGC 4147 (Brosche et al. 1985). Below we list orbital parameters for both clusters along with preliminary results for NGC 6218 . The solar motion and galactic rotation from Frenk et al. (1980) and a spherically symmetrical galactic mass distribution with $M(R) \sim R$ were used.

\begin{tabular}{|c|c|c|c|c|}
\hline Cluster & $\left(\overline{(\Pi, \Theta, Z)_{G S R}}\right.$ & $\begin{array}{c}\text { Eccentricity } \\
\mathrm{e} \\
\end{array}$ & $\begin{array}{l}\text { Perigalactic } \\
\text { Distance (kpc) }\end{array}$ & $\begin{array}{l}\text { Angular Momentum } \\
\text { Parameter } \mathrm{h}\end{array}$ \\
\hline $\begin{array}{ll}\text { NGC } & 4147 \\
\text { NGC } & 5466 \\
\text { NGC } & 6218\end{array}$ & $\begin{array}{l}(163,233,153) \\
(256,-61,223) \\
(-43,110,-130)\end{array}$ & $\begin{array}{l}0.62 \\
0.82 \\
0.39\end{array}$ & $\begin{array}{r}12.2 \\
4.7 \\
2.7\end{array}$ & $\begin{array}{l}0.62 \pm 0.12 \\
0.34 \pm 0.11 \\
0.52 \pm 0.16\end{array}$ \\
\hline
\end{tabular}

Innanen et al. (1983) suggested a lack of high-eccentricity globular cluster orbits. They found a lower limit of 0.55 for the dimensionless angular momentum $h$. Our values for $h$ are near or below this limit. In addition, we find high orbital eccentricities for the three clusters, whose common feature is their relative looseness. NGC 5466 is an example for a globular cluster in a retrograde orbit.

\section{SPACE VELOCITIES FOR 47 TUC AND NGC 362}

The galactic globular clusters 47 Tuc and NGC 362 appear projected onto the SMC. This provides the opportunity to relate directly the motions of the two clusters to that of the SMC, whose proper motion (predicted to be $0 .: 2 / 100$ yr by Lin et al. 1982) will be measured by HIPPARCOS. Firstepoch plates taken with the 13"- and 24"-refractors of the Harvard Southern Station on loan from the Harvard Plate Collection were measured with the PDS 2020GM-Microdensitometer of the Münster Astronomical Institute. All objects in the cluster fields down to the limiting magnitude of $17^{\mathrm{m}}$ were registered and are currently reduced.

\section{ACKNOWLEDGEMENTS}

We are very grateful to $\mathrm{Dr}$. A.R. Klemola for providing us with results from the Lick program prior to publication. H.-J. Tucholke thanks Dr. M. Hazen for her cooperation in the use of the Harvard Plate Collection.

\section{REFERENCES}

Brosche, P., Geffert, M., Klemola, A. R. and Ninkovic, S. 1985 Astron. J. 90, 2033.

Brosche, P., Geffert, M. and Ninkovic, S. 1983 Publ. Astron. Inst. Czech. Acad. Sci. 56, 145.

Brosche, P. and Schwan, H. 1981 Astron. Astrophys. 99, 311.

Brosche, P. and Schwan, H. 1986 in IAU Symposium No. 109, Astrometric Techniques, H. Eichorn and R. J. Leacock, eds., Reidel, Dordrecht, p. 53 .

Frenk, C. S. and White, S. D. M. 1980 Monthly Notices Roy. Astron. Soc. $193,295$.

Innanen, K. A., Harris, W. E. and Webbink, R. F. 1983 Astron. J. 88,338 .

Lin, D. N. C. and Lynden-Bell, E. 1982 Monthly Notices Roy. Astron. Soc. 198,707 . 